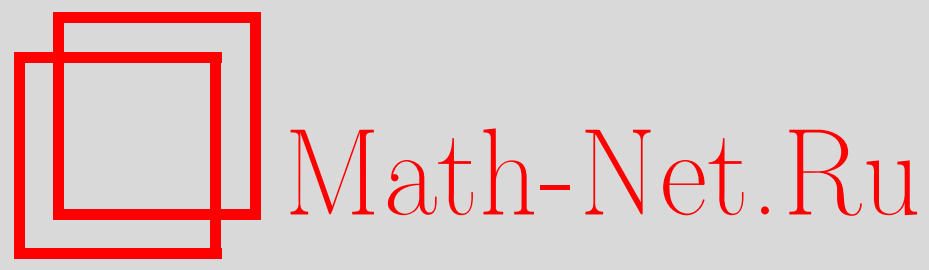

А. В. Карабегов, Строгое деформационное квантование на псевдокэлеровой орбите компактной группы Ли, Функи. анализ и его прил., 1998, том 32 , выпуск 1, 66-68

DOI: https://doi.org/10.4213/faa400

Использование Общероссийского математического портала MathNet.Ru подразумевает, что вы прочитали и согласны с пользовательским соглашением http://www . mathnet.ru/rus/agreement

Параметры загрузки:

IP: 54.81 .137 .203

26 апреля 2023 г., 13:15:37

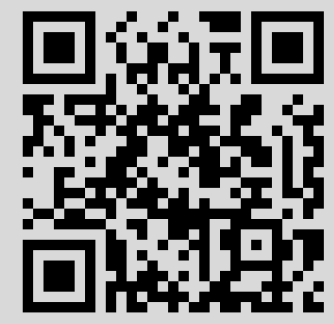




\title{
Строгое деформационное квантование на псевдокэлеровой орбите компактной группы Ли
}

\author{
(C) 1998. А. В. КАРАБЕГОВ
}

В данной заметке мы строим так называемое строгое деформационное квантование (см. [1]) на орбите компактной полупростой группы Ли, на которой задана инвариантная псевдокэлерова поляризация, пользуясь результатами, полученными в [2]. Деформационное квантование на симплектическом многообразии $M$ (см. [3]) задается ассоциативным умножением $\star$ в формальных рядах $C^{\infty}(M)[[\nu]], f \star g=\sum_{r} \nu^{r} C_{r}(f, g)$. Здесь $C_{r}$ - бидифференциальные операторы, причем $C_{0}(f, g)=f g, C_{1}(f, g)-C_{1}(g, f)=i\{f, g\}$, где $\{\cdot, \cdot\}$ - скобка Пуассона на $M$. Деформационное квантование называется строгим, если на подходящем классе функций на $M$ задано зависящее от параметра $\hbar$ семейство ассоциативных умножений $\{* \hbar\}$, такое, что произведение функций $f *_{\hbar} g$ разлагается в абсолютно сходящийся ряд Тейлора по $\hbar$ в окрестности нуля, $f *_{\hbar} g=\sum_{r} \hbar^{r} C_{r}(f, g)$.

1. Пусть $K$ - вещественная группа Ли, $k_{r}$ - ее алгебра Ли, $k_{r}^{*}$ - дуальное пространство к $k_{r}$ и $\Omega \subset k_{r}^{*}$ - коприсоединенная орбита, на которой задана инвариантная псевдокэлерова поляризация. Таким образом, форма Кириллова $\omega$ на орбите будет формой типа $(1,1)$ относительно комплексной структуры, определяемой поляризацией. Каждому элементу $X \in k_{r}$ отвечает фундаментальное векторное поле $v_{X}$ на $\Omega$, гамильтоново относительно формы Кириллова, и гамильтониан $f_{X}$ этого поля задается формулой $f_{X}(F)=\langle F, X\rangle, F \in \Omega$. Пусть $v_{X}=\xi_{X}+\eta_{X}$ - разложение поля $v_{X}$ на компоненты типа $(1,0)$ и $(0,1)$ соответственно. Поскольку комплексная структура $K$-инвариантна, поле $\xi_{X}$ голоморфно, а $\eta_{X}$ антиголоморфно. Для $X \in k_{r}$ введем дифференциальные операторы $l_{X}^{(\hbar)}=\xi_{X}+(i / \hbar) f_{X}$ и $r_{X}^{(\hbar)}=\eta_{X}-(i / \hbar) f_{X}$ на $\Omega$.

ПредлОЖенИЕ 1. Отображения $k_{r} \ni X \mapsto l_{X}^{(\hbar)} u X \mapsto r_{X}^{(\hbar)}$ являются гомоморфизмами алгебры Ли $k_{r}$ в алгебру Ли дифференциальных операторов первого порядка на $\Omega$. Для любых $X, Y \in k_{r}$ операторы $l_{X}^{(\hbar)}$ u $r_{Y}^{(\hbar)}$ коммутируют.

Пусть $g_{c}=k_{r} \otimes \mathbb{C}$ - комплексификация алгебры Ли $k_{r}$ и $\mathscr{U}\left(g_{c}\right)-$ универсальная обертывающая алгебра алгебры Ли $g_{c}$. Продолжим $X \mapsto l_{X}^{(\hbar)}$ и $X \mapsto r_{X}^{(\hbar)}$ до коммутирующих представлений $\mathscr{U}\left(g_{c}\right) \ni u \mapsto l_{u}^{(\hbar)}$ и $u \mapsto r_{u}^{(\hbar)}$ алгебры $\mathscr{U}\left(g_{c}\right)$ дифференциальными операторами на $\Omega$.

Пусть $u \mapsto \check{u}$ - антиавтоморфизм алгебры $\mathscr{U}\left(g_{c}\right)$, такой, что $\check{X}=-X$ для $X \in g_{c}$.

Лемма 1. Для $u \in \mathscr{U}\left(g_{c}\right)$ выполняется равенство $l_{u}^{(\hbar)} 1=r_{\check{u}}^{(\hbar)} 1$.

Введем отображение $\mathscr{U}\left(g_{c}\right) \ni u \mapsto s_{\hbar} u=l_{u}^{(\hbar)} 1$. Из леммы 1 следует, что ядро $I$ этого отображения является двусторонним идеалом в $\mathscr{U}\left(g_{c}\right)$. Обозна- 
чим через $\mathscr{A}_{\hbar}$ образ отображения $u \mapsto s_{\hbar} u$. На $\mathscr{A}_{\hbar}$ возникает ассоциативное умножение $* \hbar$, индуцированное с факторалгебры $\mathscr{U}\left(g_{c}\right) / I$. Отметим, что для $u, v \in \mathscr{U}\left(g_{c}\right)$ выполняются соотношения $l_{u}^{(\hbar)} s_{\hbar} v=s_{\hbar} u *_{\hbar} s_{\hbar} v=s_{\hbar}(u v)$.

2. В [4] мы построили деформационное квантование на произвольном кэлеровом многообразии. Эта конструкция тривиальным образом обобщается на псевдокэлеровы многообразия.

Операторы левого и правого ћ-умножений деформационного квантования являются формальными рядами дифференциальных операторов, или, коротко, формальными дифференциальными операторами.

ПРЕДЛОЖЕНИЕ 2. На произвольном псевдоћэлеровом многообразии $M c$ псевдокэлеровой формой $\omega$ существует единственное деформачионное квантование $\left(C^{\infty}(M)[[\nu]], \star\right)$, такое, что алгебра $\mathscr{L}$ формальньх дифференииальных операторов левого ћ-умножения на $M$ полностью определяется следуюшим условием: Формальный дифференциальный оператор на $\Omega$ принадлежит $\mathscr{L}$, если его ограничение на любую стягиваемую координатную окрестность $U \subset M$ с голоморфньли координатами $z^{k}$ и локальным потенциалом $\Phi$ формь $\omega$ коммутирует с операторами умножения на $\bar{z}^{l}$ и с $\partial \Phi / \partial \bar{z}^{l}+$ $\nu \partial / \partial \bar{z}^{l}$.

Заметим, что ^-умножение непосредственно распространяется на пространство формальных рядов Лорана с конечной главной частью $\mathscr{F}=$ $\left.C^{\infty}(M)\left[\nu^{-1}, \nu\right]\right]$.

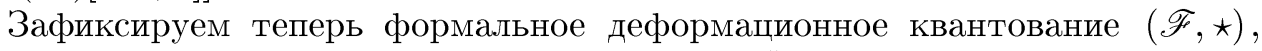
введенное в предложении 2 , на псевдокэлеровой орбите $\Omega$. Для $X \in k_{r}$ зададим формальный дифференциальный оператор $l_{X}^{(\nu)}=\xi_{X}+(i / \nu) f_{X}$. Отображение $k_{r} \ni X \mapsto l_{X}^{(\nu)}$ является гомоморфизмом в алгебру Ли формальных дифференциальных операторов первого порядка на $\Omega$ и продолжается до гомоморфизма алгебры $\mathscr{U}\left(g_{c}\right) \ni u \mapsto l_{u}^{(\nu)}$ в алгебру формальных дифференциальных операторов на $\Omega$.

ПРЕДЛОЖЕНИЕ 3. Для $u \in \mathscr{U}\left(g_{c}\right)$ оператор $l_{u}^{(\nu)}$ принадлежит алаебре операторов левого ћ-умножения $\mathscr{L}$. Таким образом, отображение $\mathscr{U}\left(g_{c}\right) \ni$ $u \mapsto l_{u}^{(\nu)} 1$ является гомоморфизмом алгебры $\mathscr{U}\left(g_{c}\right)$ в алгебру $(\mathscr{F}, \star)$.

3. Пусть теперь $K$ - компактная полупростая группа Ли. Псевдокэлеровы поляризации на орбитах группы $K$ легко описать явно. Они связаны с реализацией орбит в виде $G / P$, где $G$ - комплексная полупростая группа Ли, в которой $K$ - максимальная компактная подгруппа, а $P$ - параболическая подгруппа. Можно показать, что алгебры функций на коприсоединенной орбите $\Omega$ группы $K$, введенные в [2], совпадают с алгебрами $\mathscr{A}_{\hbar}$, построенными по некоторой псевдокэлеровой поляризации на $\Omega$. В частности, когда форма $(1 / \hbar) \omega$ на орбите $\Omega$ целочисленна и кэлерова, то алгебра $\mathscr{A}_{\hbar}$ совпадает с алгеброй ковариантных символов Березина на кэлеровом многообразии $(\Omega,(1 / \hbar) \omega)$ (см. [5]).

Введем на $\mathscr{U}\left(g_{c}\right)$ фильтрацию $\left\{\mathscr{U}_{d}\right\}$, где $\mathscr{U}_{d}$ порождена мономами вида $X_{1} \cdots X_{k}, k \leqslant d, X_{j} \in g_{c}$. Для $u \in \mathscr{U}_{d}$ функция $s_{\hbar} u$ является многочленом 
от $1 / \hbar$ степени не выше $d$. Напомним, что функция на орбите $\Omega$, являющаяся ограничением многочлена, заданного на $k_{r}^{*}$, называется регулярной. Следуюшее предложение доказано в работе [2].

ПРЕДЛОЖЕНИЕ 4. Для яюбой регулярной функиии $f$ на орбите $\Omega$ существуют такие элементьл $u_{j} \in \mathscr{U}_{d(j)}$ и рачиональнье функции $a_{j}(\hbar)$, не имеющие полюса в нуле, что $f=\sum_{j} a_{j}(\hbar) \hbar^{d(j)} s_{\hbar} u_{j}$ при всех, кроме конечного числа, значениях $\hbar$.

Из этого предложения следует, что регулярная функция на орбите $\Omega$ принадлежит алгебре $\mathscr{A}_{\hbar}$ для всех, кроме конечного числа, значений $\hbar$. Более того, для любых двух регулярных функций $f, g$ на $\Omega$ и $F \in \Omega$, произведение $f *_{\hbar} g(F)$ рационально по $\hbar$ и не имеет полюса в нуле. Пользуясь соображениями из [6], получаем, что произведение $f * \hbar g$ разлагается в абсолютно сходящийся ряд Тейлора по $\hbar$ в окрестности нуля. Обозначим соответствуюший формальный асимптотический ряд через $f *_{\nu} g$. Пусть при этом $f=\sum_{j} a_{j}(\hbar) \hbar^{d(j)} s_{\hbar} u_{j}$. Тогда $f *_{\hbar} g=\sum_{j} a_{j}(\hbar) \hbar^{d(j)} l_{u_{j}}^{(\hbar)} g$. Обозначим через $a_{j}(\nu)$ формальное асимптотическое разложение функции $a_{j}(\hbar)$ в нуле (т.е. ряд Тейлора, в котором переменная $\hbar$ заменена на формальный параметр $\nu)$. Тогда $f *_{\nu} g=\sum_{j} a_{j}(\nu) \nu^{d(j)} l_{u_{j}}^{(\nu)} g$. Из того что $\sum_{j} a_{j}(\nu) \nu^{d(j)} l_{u_{j}}^{(\nu)} \in \mathscr{L}$, непосредственно следует, что $f *{ }_{\nu} g=f \star g$.

ТЕОРемА. На любой орбите $\Omega$ компактной полупростой группьл Ли $K$, на которой зафиксирована инвариантная псевдокэлерова поляризачия, сушествует семейство ассоииативных алаебр $\left(\mathscr{A}_{\hbar}, *_{\hbar}\right)$, элементами которых являютсл регулярнье функиии. Любая регулярная функиия на $\Omega$ принадлежит $\mathscr{A}_{\hbar}$ для всех, кроме конечного числа, значений $\hbar$. Для любьх регулярньх функиий $f, g$ на $\Omega$ произведение $f *_{\hbar} g$ разлагается в абсолютно сходящийся ряд Тейлора по $ћ$ в окрестности нуля. Этот ряд определяется

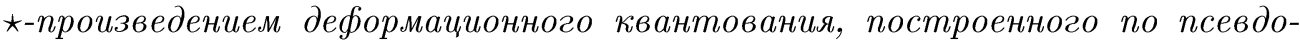
кэлеровой структуре на $\Omega$.

Таким образом, на псевдокэлеровых орбитах компактной полупростой группы Ли возникает строгое деформационное квантование. Этот факт был установлен в [6] для кэлеровых орбит, являющихся компактными эрмитовыми симметрическими пространствами и высказан в качестве гипотезы для общих кэлеровых орбит.

Автор выражает благодарность за оказанное гостеприимство Международному центру по теоретической физике, Триест, Италия, где была выполнена часть работы.

\section{ЛИТЕРАТУРА}

1. Weinstein A. Astérisque, 227, 389-409 (1995). 2. Karabegov A. V. Trans. Am. Math. Soc. (1998), to appear. 3. Bayen F. et al. Ann. Phys., 111, No. 1, 1-151 (1978). 4. Карабегов А. В. Функц. анализ и его прил., 30, вып. 2, 87-89 (1996). 5. Березин Ф. А. Изв. АН СССР, сер. матем., 38, 1116-1175 (1974). 6. Cahen M., Gutt S., Rawnsley J. H. Trans. Am. Math. Soc., 337, No. 1, 73-98 (1993). 